\title{
Electroencephalogram Analysis Based on Gramian Angular Field Transformation
}

\author{
A.D. Bragin ${ }^{1}$, V.G. Spitsyn ${ }^{1}$ \\ lflenylol@gmail.com|spvg@tpu.ru \\ ${ }^{1}$ National Research Tomsk Polytechnic University, Tomsk, Russia
}

\begin{abstract}
This paper addresses the problem of motion imagery classification from electroencephalogram signals which related with many difficulties such on human state, measurement accuracy, etc. Artificial neural networks are a good tool to solve such kind of problems. Electroencephalogram is time series signals therefore, a Gramian Angular Fields conversion has been applied to convert it into images. GAF conversion was used for classification EEG with Convolutional Neural Network (CNN). GAF images are represented as a Gramian matrix where each element is the trigonometric sum between different time intervals. Grayscale images were applied for recognition to reduce numbers of neural network parameters and increase calculation speed. Images from each measuring channel were connected into one multi-channel image. This article reveals the possible usage GAF conversion of EEG signals to motion imagery recognition, which is beneficial in the applied fields, such as implement it in brain-computer interface
\end{abstract}

Keywords: motor imagery recognition, electroencephalogram, Gramian Angular Field, Convolutional Neural Network.

\section{Introduction}

Electroencephalography is one of the most popular noninvasive methods for studying brain activity today. Signals of the electroencephalogram (EEG) show the total electrical activity of neurons in the cerebral cortex, studying these data, you can get a lot of useful information about the human condition.

The study of EEG is associated with many difficulties, such as the dependence of signals on age, time of day, the presence of noise, interference and a weak degree of structure.

Classical mathematics methods based on time-frequency, wave or component analysis can be used to study EEG. However, their application often does not provide stable results of recognition of various human conditions, and in some cases their application becomes extremely difficult due to the complexity of the algorithms $[2,4,6,7]$. Signals of the brain are very complex, which is the main cause of this problem. Classical mathematical techniques (Fourier transforms, wavelet analysis, etc.) are based on the selection of a useful signal from the entire data array and further algorithmic work with it. Often, the selection of such a useful signal is difficult for signals recorded in difficult conditions of psychophysiological experiments, and with the slightest change in state the technique may stop working.

The use of artificial neural networks (ANNs) in application areas such as the brain-computer interface is today a promising area of research $[5,9,13]$. The ability of ANNs to adaptive learning, resistance to signal distortion and a good generalizing effect makes them an excellent tool for classification $[11,12,16]$.

There are several approaches to the classification of time series using ANNs [10]. A key factor in the success of the recognition of human activities using EEG is the effective use of data obtained from measuring sensors. In this paper, we use the method proposed in [16]. In this method, the time series is converted into images, after which a convolutional neural network $(\mathrm{CNN})$ is used to analyze them.

\section{GAF transform}

The GAF (Gramian Angular Field) method [15] was used to classify EEG signals using convolutional neural networks. In this method, the time series is converted to a polar coordinate system. The matrix $G$ is built on the basis of the data obtained, each element of the matrix is equal to the cosine of the sum of the angles. The resulting matrix is converted into an image, which is fed to the input of the convolutional neural network.

The preservation of the time dependence is ensured by such a transformation. The main diagonal is a special case at $\mathrm{k}=0$, containing the initial values and angular information.

The GAF matrix is a matrix constructed in a series as follows:
1. First, the row is normalized to a segment $[-1,1]$ :

$$
\hat{x}_{i}=\frac{\left(x_{i}-\max (X)\right)+\left(x_{i}-\min (X)\right)}{\max (X)-\min (X)} .
$$

2. Further, the obtained values are translated into the polar coordinate system as follows:

$$
\left\{\begin{array}{c}
\varphi_{i}=\arccos \left(x_{i}\right) \\
r_{i}=\frac{\mathrm{t}_{\mathrm{i}}}{\mathrm{N}}
\end{array} .\right.
$$

3. The GAF matrix is calculated by the formula:

$$
G=\left[\begin{array}{ccc}
\cos \left(\varphi_{1}+\varphi_{1}\right) & \cdots & \cos \left(\varphi_{1}+\varphi_{n}\right) \\
\cos \left(\varphi_{2}+\varphi_{1}\right) & \cdots & \cos \left(\varphi_{2}+\varphi_{n}\right) \\
\vdots & \ddots & \vdots \\
\cos \left(\varphi_{n}+\varphi_{1}\right) & \cdots & \cos \left(\varphi_{n}+\varphi_{n}\right)
\end{array}\right] .
$$

The final matrix stores all the information about the series, except for the initial boundaries of the values that we lose in step (1) after the normalization procedure - that is, we can restore the original series from the matrix obtained, but only scaled to the interval $[-1,1]$.

Based on the obtained matrices, images are formed for further use (Fig. 1). The work uses single-color images, since color channels in this case do not carry useful information. This allowed to reduce the number of image channels by 3 times compared with the RGB option.

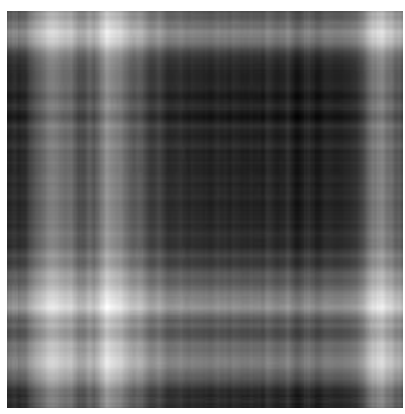

Fig. 1. GAF image example

\section{Deep convolutional neural network architecture}

In this work, the deep CNN architecture was developed, adapted to classify an EEG signal taking into account the number of measuring electrodes. Input images are fed to the input of the network in the form of a 64-channel image, where each channel is a transformed electroencephalogram signal. The architecture 
of the deep CNN consists of the main layers: three convolutional and three fully connected. Network parameters were determined by the selection method.

\section{Used data}

The data presented in [3] were selected for research. Each subject was in a chair with armrests and watched the image on the monitor. At the beginning of each test, the monitor displayed a black screen with a fixing cross for two seconds, then the subject had to imagine the movement of the hand depending on the instructions on the monitor for three seconds. After which there was a short break for several seconds, upon its completion the action was repeated.

The data set is EEG signals recorded using the BCI 2000 system [1] and using 64 electrodes at a sampling frequency of $512 \mathrm{~Hz}$. Frequency filters for data conversion were not used.

The order of the experiment and the conversion of the source data into GAF images are presented in Fig. 2. For the training of neural networks, the data of the first subject were used.

\section{Black screan}

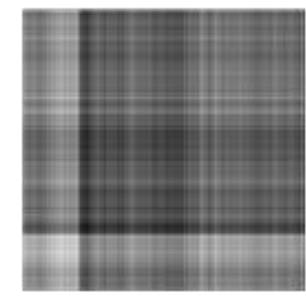

$\begin{array}{llllll} & \mid & \mid & & \mid & \\ -2 & -1 & 0 & 1 & 2 & \text { sec }\end{array}$

Fig. 2. Experiment order

\section{Model structure}

Electroencephalograms are signals received from 64 measuring channels. Each signal is converted to a separate image using GAF conversion. The generated images are input to the model. By analogy with the RGB representation, the resulting GAF images are converted into an input vector, however, if 3 channels are used in the case of the RGB image, one for each color, in this case a 64 channel image containing the converted EEG signal is input. The structural scheme of the final model is presented in Fig. 3.

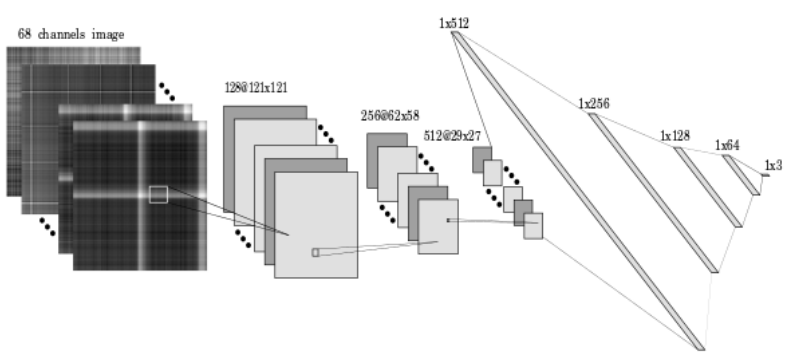

Fig. 3. Model structure

\section{Motor imagery recognition}

The data of the first subject [17] were used to recognize motor imagery. Images 128 by 128 pixels in size were fed to the input of a neural network. Recognition accuracy was about 97\%, with an accuracy of $80 \%$ from the data source.

\section{Conclusion}

The article examined the possibility of using the GAF conversion method for detecting motor imageries in EEG signals. Without the use of additional filtration, high results can be achieved. The recognition accuracy of motor imageries of movement with the right and left hand and the state of rest was 97\% for the studied EEG signals. General results indicate that this method gives higher accuracy than the methods described in the data source. The presented method of classification of electroencephalograms can be used to build a brain-computer interface.

\section{Acknowledgments}

The reported study was funded by RFBR according to the research project № 18-08-00977 A.

\section{References}

[1] Blankertz B. et al The BCI competition 2003: Progress and perspectives in detection and discrimination of EEG single trials// IEEE Transactions on Biomedical Engineering. 2004. - №6 (51). - P. 1044-1051.

[2] Grubov V.V., Runnova A.E., Kurovskaya M.K., Pavlov A.N., Koronovskii A.A., Hramov A.E. Demonstration of brain noise on human EEG signals in perception of bistable images // Proc. SPIE. 2016. V. 9707. DOI: $10.1117 / 12.2207390$

[3] Hohyun Cho, Minkyu Ahn, Sangtae Ahn, Moonyoung Kwon, Sung Chan Jun, EEG datasets for motor imagery brain-computer interface, GigaScience, Volume 6, Issue 7 , July 2017, gix034, https://doi.org/10.1093/gigascience/gix034

[4] Hramov A.E., Koronovskii A.A., Makarov V.A., Pavlov A.N., Sitnikova E.Y. Wavelets in Neuroscience. Heidelberg; New York; Dordrecht; London, Springer, 2015. $318 \mathrm{p}$

[5] W. Hsu and I. Chiang, "Application of neural network to brain-computer interface," 2012 IEEE International Conference on Granular Computing, Hangzhou, 2012, pp. 163-168. doi: 10.1109/GrC.2012.6468559

[6] Koronovskii A.A., Hramov A.E., Grubov V.V., Moskalenko O.I., Sitnikova E.Y., Pavlov A.N. Coexistence of intermittencies in the neuronal network of the epileptic brain // Phys. Rev. E. 2016. V. 93. DOI: 10.1103/PhysRevE.93.032220

[7] van Luijtelaar G., Lüttjohann A., Makarov V.V., Maksimenko V.A., Koronovskii A.A., Hramov A.E. Methods of automated absence seizure detection, interference bystimulation, and possibilities for prediction in genetic absence models // Journal of Neuroscience Methods. 2016. V. 260. P. 144.

[8] Maksimenko V.A., Heukelum S., Makarov V.V., Kelderhuis J., Lüttjohann A., Koronovskii A.A., Hramov A.E., Luijtelaar G. Absence Seizure Control by a Brain Computer Interface // Scientific Reports. 2017. V. 7. P. 2487.

[9] K. Nakayama and K. Inagaki, "A Brain Computer Interface Based on Neural Network with Efficient Pre-Processing," 2006 International Symposium on Intelligent Signal Processing and Communications, Tottori, 2006, pp. 673676. doi: 10.1109/ISPACS.2006.364745

[10] Nima Hatami, Yann Gavet, Johan Debayle. Classification of Time-Series Images Using Deep Convolutional Neural Networks. 2017 The 10th International Conference on 
Machine Vision (ICMV 2017), ICMV Committees, Nov 2017, Vienne, Austria. 10.1117/12.2309486. hal-01743695

[11] R. Östberg, 'Robustness of a neural network used for image classification: The effect of applying distortions on adversarial examples', Dissertation, 2018.

[12] Qinglong Wang, Wenbo Guo, Kaixuan Zhang, Alexander G. Ororbia, II , Xinyu Xing , Xue Liu , C. Lee Giles, Adversary Resistant Deep Neural Networks with an Application to Malware Detection, Proceedings of the 23rd ACM SIGKDD International Conference on Knowledge Discovery and Data Mining, August 13-17, 2017, Halifax, NS, Canada

[13] Vasilyev A.N., Liburkina S.P., Kaplan A.Y. Lateralization of EEG patterns in humans during motor imagery of arm movements in the brain-computer interface // Zhurnal Vysshei Nervnoi Deyatelnosti Imeni IP Pavlova. 2016. V. 66. № 3. P. 302-312.

[14] Wang, Z.; Oates, T. Imaging time-series to improve classification and imputation. In Proceedings of the Twenty-Fourth International Joint Conference on Artificial Intelligence, Buenos Aires, Argentina, 25-31 July 2015; pp. 3939-3945.

[15] Wang, Z., Oates, T. Encoding time series as images for visual inspection and classification using tiledconvolutional neural networks, Association for the Advancement of Artificial Intelligence (AAAI) conference,2015.

[16] J. Yim and K. Sohn, "Enhancing the Performance of Convolutional Neural Networks on Quality Degraded Datasets," 2017 International Conference on Digital Image Computing: Techniques and Applications (DICTA), Sydney, NSW, 2017, pp. 1-8. doi: 10.1109/DICTA.2017.8227427 Nima Hatami, Yann Gavet, Johan Debayle.

[17] Zhiguang Wang и Tim Oates. "Spatially Encoding Temporal Correlations to Classify Temporal Data Using Convolutional Neural Networks". B: CoRR abs/1509.07481 (2015). url: http://arxiv.org/abs/1509.07481. 